\title{
Two types of geomagnetic storms and relationship between Dst and $A E$ indexes
}

\author{
Lyudmila P. Shadrina ${ }^{1, *}$ \\ ${ }^{1}$ Academy of sciences of Sakha (Yakutia) Republic, Yakutsk, Russia
}

\begin{abstract}
The study of the relationship between Dst and AE indices of the geomagnetic field and its manifestation in geomagnetic storms in the XXIII solar cycle was carried out. It is shown that geomagnetic storms are divided into two groups according to the ratio of the amplitude of Ds index decrease to the sum of the $\mathrm{AE}$ index during the main phase of the storm. For the first group it is characteristic that for small depressions of the Dst index, significant amounts of the $\mathrm{AE}$ index are observed. Most often these are storms with a gradual beginning and a long main phase associated with recurrent solar wind streams. Storms of the second group differ in large amplitudes of Dst index decrease, shorter duration of main phase and small amounts of AE-index. Usually these are sporadic geomagnetic storms with a sudden commencement caused by interplanetary disturbances of the CME type. The storms of these two types differ also in their geoeffects, including the effect on human health.
\end{abstract}

\section{Introduction}

In this paper, the analysis of geomagnetic storms from 1996 to 2007, during the XXIII solar cycle was fulfilled. This solar cycle was actively discussed in the literature [1-11] mainly because unusually intense geomagnetic storms with Dst index decreases more than $400 \mathrm{nT}$ during the maximum of the cycle (2000-2004) were observed. All of them are devoted to a comprehensive study of these storms - the analysis of solar phenomena, the identification of interplanetary perturbations, ground manifestations in the geomagnetic field, in optical phenomena, propagation of waves, etc. The main attention was paid to studies of extra storms in 2001, 2003 and 2004, and only in [10] the moderate storms were considered. In contrast to these works here are the results of a statistical analysis of geomagnetic disturbances from the point of view of dividing storms into two types - sporadic and recurrent - and not only and not so much intense and extra storms but moderate and weak one are studies.

It is known that sporadic storms are associated with the passage of solar wind disturbed structures, caused mainly by solar flares, with the propagation of coronal mass ejections (CME). The reason for the second type of storms are recurrent high-speed fluxes from coronal holes [8, 9, 11]. So, usually the division of storms into two types is determined mainly by the recurrence of events: geomagnetic storms refer to recurrent ones, if they are repeated with a periodicity of 27 days - the time of rotation of the Sun around its axis.

\footnotetext{
* e-mail: lushadr@mail.ru
} 


\section{Data}

In papers [12-14] it was proposed to determine the type of storm using the ratio of ground based indices of geomagnetic activity - $\mathrm{AE}$ and Dst. The basis for determining the magnitude of these indices is the horizontal $\mathrm{H}$ component of the geomagnetic field. To calculate the AE index, magnetograms of the $\mathrm{H}$ components of 12 observatories uniformly distributed over the longitude in the northern hemisphere at auroral or subauroral latitudes, between $60^{\circ}$ and $70^{\circ}$ are used. The AE index is defined as the difference between the AL and AU indices (the largest negative and positive variations of the $\mathrm{H}$ component) and characterizes the intensity of the auroral zone currents (auroral electrojet). In a quiet time, the value of this index is tens of nT, and during storms and substorms it increases to several hundred and more than a thousand nT. It was shown in $[14,15]$ that for two types of storms there is a difference in the behavior of auroral activity: during storms of sporadic type, a chain of substorms with a sharp start and a short duration are observed. The depth of modulation of the AE-index is average, up to several hundred nT, there are few intensifications. During recurrent storms, a high level of background perturbations is observed, frequent and intense surges of the AL-index lasting several minutes and a small depth of modulation of the AE-index.

The Dst-index is an axisymmetric component of the disturbed magnetic field relative to the geomagnetic dipole and is determined on the basis of measurements of the magnetic field at four equatorial stations. Its decrease by $30 \mathrm{nT}$ and more, up to $400 \mathrm{nT}$ is a manifestation of the geomagnetic storm, its main phase. According to the magnitude of this decrease storms are dividing into weak ones (30-50 nT), moderate (50-100 nT), strong (100-200 nT) and extra storms (more than $200 \mathrm{nT}$ ) [5]. The main reason for this variation is the development of a ring current located at a distance of 2-6 Earth radii due to the injection of charged particles with an energy of 1-300 $\mathrm{KeV} /$ nucleon from the tail of the magnetosphere.

The amplitude of Dst-index decrease for the storm main phase is proportional to the number of particles injected into the ring current, and thus is a measure of ring current increase, a measure of the energy input to the interior of the magnetosphere during a storm. The energy characteristic of auroral activity is the sum of the AE index over the same time interval $[16,17]$. On this basis, the parameter $\boldsymbol{k}$ is proposed in [12], which is calculated by the formula: $\boldsymbol{k}=\Delta \mathrm{Dst} / \Sigma \mathrm{AE}$, where $\Sigma$ AE is the sum of the $\mathrm{AE}$ index during the main phase of the storm $\mathrm{T}$, and $\Delta \mathrm{Dst}$ is the amplitude of the decrease of the Dst index, the difference between the maximum and minimum value of Dst for the same time interval. It is shown in [12-14] that the parameter $\boldsymbol{k}$ differs significantly for two types of storms. Storms of sporadic (flare) type have $\boldsymbol{k}<0.010$, for recurrent storms $\boldsymbol{k}>0.015$. Thus, the value of k suggests to determine the type of storm - sporadic or recurrent.

\section{Results and discussion}

The result of comparison of statistical characteristics of the two types of storms during 12 years of the XXIII solar cycle are shown in Table 1. In the first column there are Wolf numbers - the number of sunspots characterizing solar activity. One can see the maximum cycle occurs in 2000 and remains high for three years, until 2002. The type of storm was determined by the parameter $\boldsymbol{k}$. For the sporadic storms its annual average varies from 0,0151 to 0,0260 and has a mean value 0.0199 , for the recurrent ones - from 0,0064 to 0,0081 and a mean value is 0.0076 . The duration of the main phase of sporadic and recurrent storms (Ts and Tr) is significantly different, 10,0 and 16,2 hours, respectively. The average storm intensity for sporadic type is noticeably higher than for recurrent one (respectively, $83.3 \mathrm{nT}$ and $54.7 \mathrm{nT}$ ). Auroral activity is almost twice as high for recurrent storms $(\Sigma \mathrm{AE}=7676 \mathrm{nT})$ than for sporadic ones $(\Sigma \mathrm{AE}=4454 \mathrm{nT})$. So, the division of storms on two types 
according to the parameter $\mathrm{k}$ made it possible to pick out different quantitative characteristics of the storms.

Table 1. Characteristics of the geomagnetic storms in XXIII solar cycle

\begin{tabular}{|l|r|r|r|r|r|r|r|r|c|}
\hline \multirow{2}{*}{ Year } & \multirow{2}{*}{ Wolf } & \multicolumn{4}{|c|}{ Sporadic storms } & \multicolumn{4}{c|}{ Recurrent storms } \\
\cline { 3 - 10 } & & \multicolumn{1}{|c|}{ Ts } & $\Delta$ Dst & $\Sigma \mathrm{AE}$ & $\boldsymbol{k}$ & $\mathrm{Tr}$ & $\Delta$ Dst & $\Sigma \mathrm{AE}$ & $\boldsymbol{k}$ \\
\hline 1996 & 8,6 & 8,6 & 48,1 & 3100 & 0,0160 & 14,0 & 40,6 & 5519 & 0,0079 \\
\hline 1997 & 21,5 & 9,0 & 80,9 & 4321 & 0,0214 & 17,2 & 58,3 & 7658 & 0,0080 \\
\hline 1998 & 64,3 & 9,2 & 86,3 & 4863 & 0,0187 & 16,0 & 58,3 & 7580 & 0,0076 \\
\hline 1999 & 93,3 & 10,4 & 94,0 & 4709 & 0,0201 & 17,4 & 50,0 & 8748 & 0,0064 \\
\hline 2000 & 119,6 & 10,0 & 100,3 & 4487 & 0,0260 & 15,5 & 57,3 & 8033 & 0,0075 \\
\hline 2001 & 111,0 & 9,5 & 110,9 & 5123 & 0,0235 & 17,8 & 59,1 & 8317 & 0,0074 \\
\hline 2002 & 104,1 & 10,3 & 80,1 & 4302 & 0,0215 & 17,8 & 56,2 & 7975 & 0,0077 \\
\hline 2003 & 63,6 & 9,5 & 104,6 & 5411 & 0,0180 & 16,7 & 58,6 & 9085 & 0,0071 \\
\hline 2004 & 40,4 & 12,1 & 79,0 & 5241 & 0,0151 & 14,0 & 51,5 & 6780 & 0,0078 \\
\hline 2005 & 29,8 & 7,0 & 82,1 & 3881 & 0,0223 & 15,3 & 60,7 & 7575 & 0,0081 \\
\hline 2006 & 15,2 & 11,8 & 74,2 & 4435 & 0,0172 & 14,5 & 50,5 & 6712 & 0,0080 \\
\hline 2007 & 7,5 & 12,4 & 58,9 & 3575 & 0,0187 & 17,8 & 55,6 & 8135 & 0,0073 \\
\hline Mean & & $\mathbf{1 0 , 0}$ & $\mathbf{8 3 , 3}$ & $\mathbf{4 4 5 4}$ & $\mathbf{0 , 0 1 9 9}$ & $\mathbf{1 6 , 2}$ & $\mathbf{5 4 , 7}$ & $\mathbf{7 6 7 6}$ & $\mathbf{0 , 0 0 7 6}$ \\
\hline
\end{tabular}

Fig. 1 shows a diagram of the quantitative characteristics of storms. Curve shows solar activity (Wolf numbers), solid line - the total number of storms $(\mathrm{N})$, columns with a rare hatching - quantity of sporadic storms (Ns), with a frequent hatching - quantity of recurrent storms ( $\mathrm{Nr}$ ). In the cycle under consideration it should be noted an appearance of a well-known pattern - two maxima of geomagnetic activity - at growth phase (1998 and 2000) and at the decay phase of solar activity (2003 and 2005).

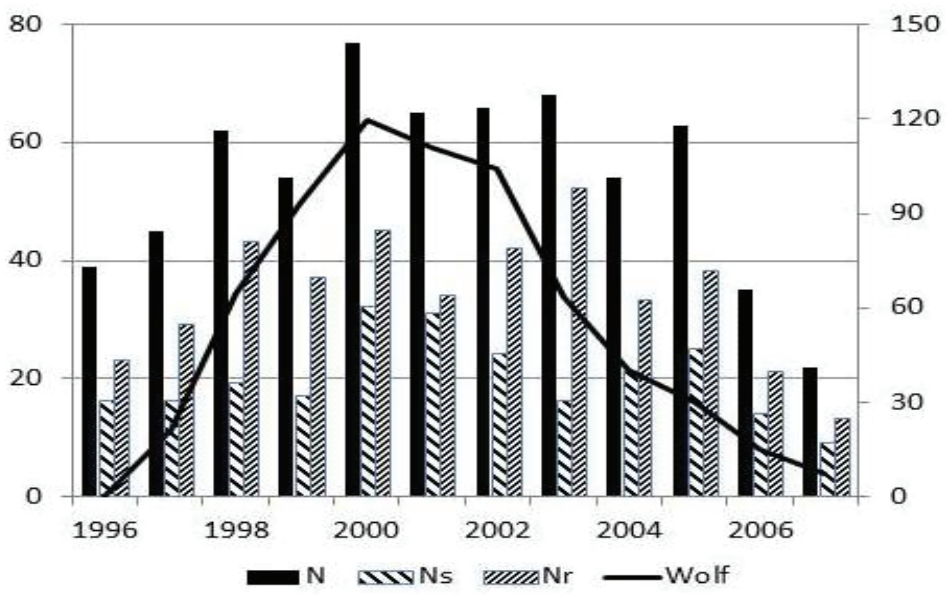

Figure 1. The total number of geomagnetic storms $(\mathrm{N})$, number of sporadic $(\mathrm{Ns})$ and recurrent $(\mathrm{Nr})$ storms in XXIII cycle of solar activity (Wolf). 
The ratio of sporadic and recurrent storms, against the expected, persists throughout the cycle: recurrent storms number are always greater than sporadic storms number (Fig. 1). Although it is generally believed that the number of sporadic storms is greater in the phase of solar activity growth, in the phase of recession - less than recurrent [10]. If do not take into account weak storms with $\Delta$ Dst $<50 \mathrm{nT}$ then in the maximum of the cycle (2000 and 2001) and in the minimum $(1996,2004-$ 2007) number of sporadic storms are greater than recurrent ones. Table 2 shows the total number of storms $(\mathrm{N})$, the number of all sporadic $(\mathrm{Ns})$ and recurrent $(\mathrm{Nr})$ storms, as well as the number of moderate, strong and extra sporadic (Ns-sm) and recurrent (Nr-sm) storms, i.e . with the exception of weak storms.

Table 2. The number of two types of storms and regression coefficients

\begin{tabular}{|c|c|c|c|c|c|c|c|c|c|}
\hline \multirow{2}{*}{ Year } & \multirow{2}{*}{$\mathrm{N}$} & \multicolumn{4}{|c|}{ Sporadic } & \multicolumn{4}{|c|}{ Recurrent } \\
\cline { 3 - 11 } & & $\mathrm{Ns}$ & Ns-sm & $\boldsymbol{a}$ & $\boldsymbol{b}$ & $\mathrm{Nr}$ & $\mathrm{Nr}-\mathrm{sm}$ & $\boldsymbol{a}$ & $\boldsymbol{b}$ \\
\cline { 3 - 11 } & 39 & 16 & 5 & 61 & 168 & 23 & 4 & 62 & 3008 \\
\hline 1997 & 45 & 16 & 12 & 37 & 969 & 29 & 14 & 72 & 3442 \\
\hline 1998 & 62 & 19 & 14 & 48 & 725 & 43 & 18 & 91 & 2290 \\
\hline 1999 & 54 & 17 & 12 & 17 & 3150 & 37 & 12 & 74 & 5060 \\
\hline 2000 & 77 & 32 & 24 & 24 & 2074 & 45 & 19 & 103 & 2454 \\
\hline 2001 & 65 & 31 & 26 & 27 & 2069 & 34 & 20 & 72 & 4041 \\
\hline 2002 & 66 & 24 & 17 & 43 & 753 & 42 & 20 & 138 & 183 \\
\hline 2003 & 68 & 16 & 11 & 34 & 1830 & 52 & 23 & 57 & 5752 \\
\hline 2004 & 54 & 21 & 15 & 25 & 2562 & 33 & 8 & 107 & 1267 \\
\hline 2005 & 63 & 25 & 19 & 21 & 2195 & 38 & 18 & 91 & 2037 \\
\hline 2006 & 35 & 14 & 11 & 55 & 364 & 21 & 7 & 117 & 799 \\
\hline 2007 & 22 & 9 & 6 & 60 & 67 & 13 & 5 & 82 & 3553 \\
\hline Mean & $\mathbf{6 5 0}$ & $\mathbf{2 4 0}$ & $\mathbf{1 7 2}$ & $\mathbf{3 7 , 7}$ & $\mathbf{1 4 1 0 , 5}$ & $\mathbf{4 1 0}$ & $\mathbf{1 6 8}$ & $\mathbf{8 8 , 8}$ & $\mathbf{2 8 2 3 , 8}$ \\
\hline
\end{tabular}

The total number of storms in this cycle was 650 , including 240 sporadic and 410 recurrent storms, i.e. $\mathrm{Nr}>\mathrm{Ns}$. If we exclude weak storms, we will get approximately equal number of two types of storms, respectively, Ns-sm $=172$ and $\mathrm{Nr}$-sm $=168$. The number of moderate and strong sporadic storms at the maximum of the cycle (2000-2001) is greater than recurrent storms number, Ns-sm $>$ Nr-sm, which agrees with the generally accepted opinion.

In paper [12] it was proposed the parameter $\boldsymbol{k}$ on the basis of the analysis of the geomagnetic data of 1967-1972. It was shown that two point clouds can be distinguished on the dot diagrams of $\Sigma$ AE versus $\Delta$ Dst, each of which has its own linear regression dependence. Dot diagrams of 19962007 (see Fig. 2) also shows two groups of points for two type of storms for every year. And for each group of points, a linear relationship $\Sigma$ AE from $\Delta$ Dst can be find. This was more clearly manifested in 1999-2006. The coefficients $\boldsymbol{a}$ and $\boldsymbol{b}$ of the linear regression dependence $\Sigma \mathrm{AE}=\boldsymbol{a} * \Delta$ Dst $+\boldsymbol{b}$ were calculated for each group of points (see Table 2). Using the solar cycle average values, we can write the regression relationships:

$$
\begin{array}{ll}
\text { Sporadic storms: } & \Sigma \mathrm{AE}=38^{*} \Delta \mathrm{Dst}+1410 \\
\text { Recurrent storms: } & \Sigma \mathrm{AE}=89 * \Delta \mathrm{Dst}+2824
\end{array}
$$


$1996 \mathrm{~N}=39$

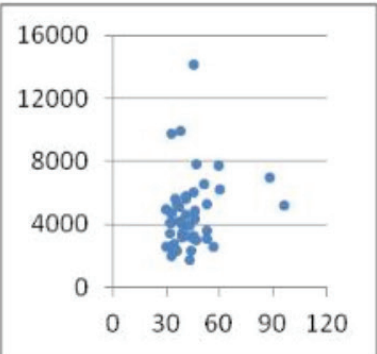

$1999 \mathrm{~N}=54$

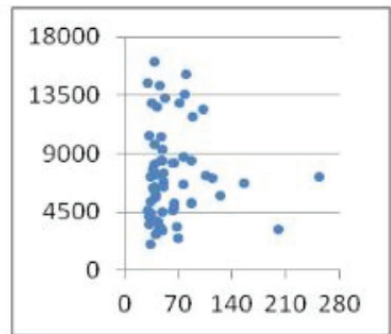

$2002 N=66$

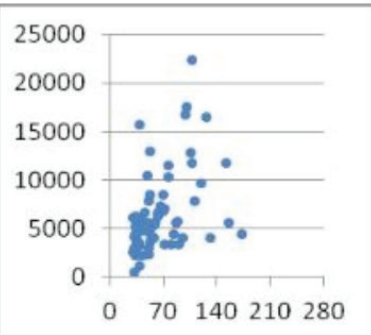

$2005 \mathrm{~N}=6$

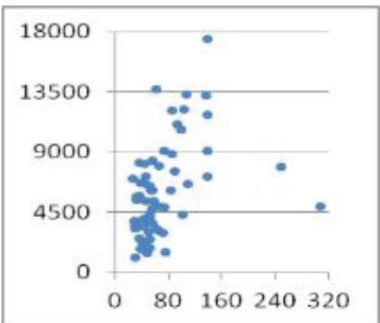

$1997 \mathrm{~N}=45$

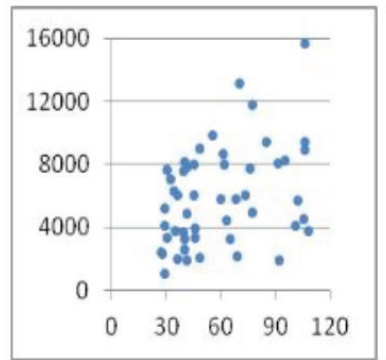

$2000 \mathrm{~N}=77$

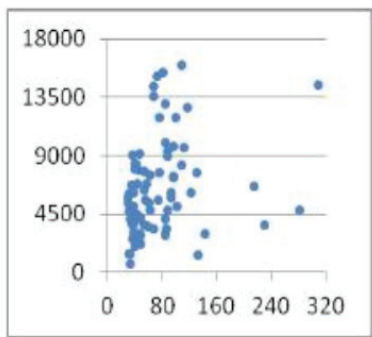

$2003 N=68$

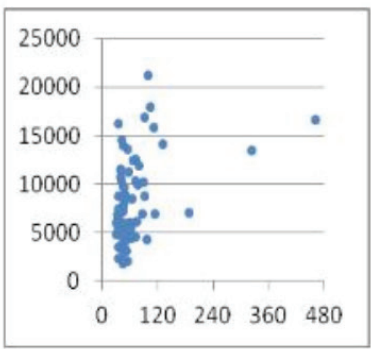

$2006 \mathrm{~N}=35$

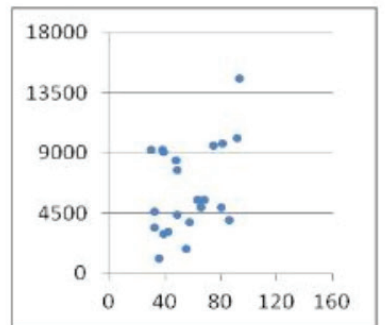

$1998 \mathrm{~N}=62$

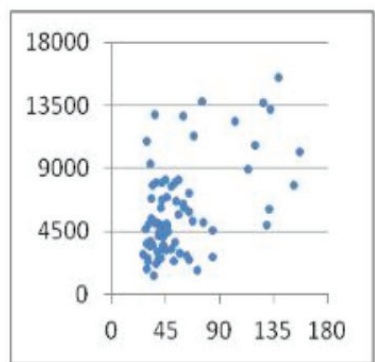

$2001 \mathrm{~N}=65$

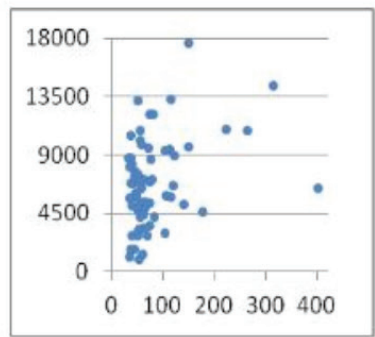

$2004 N=54$

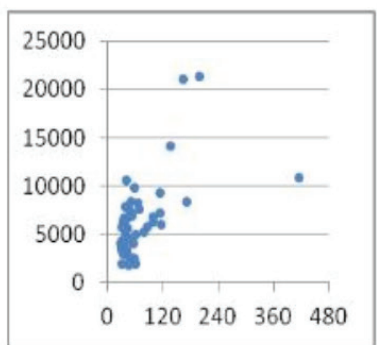

$2007 \mathrm{~N}=22$

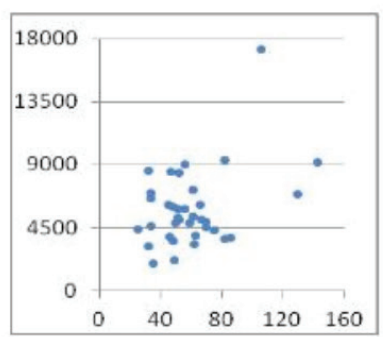

Figure 2. Point diagrams of the dependence of $\Sigma \mathrm{AE}$ (vertical) from $\Delta \mathrm{Dst}$ (horizontal). 
And one more feature of sporadic and recurrent storms - the differ in their terrestrial manifestations. In particular, it is shown in paper [18] that an increase in one of the parameter of a human cardiograms is associated with storms. During sporadic storms, the greatest number of patients with such changes occur on the first day of the geomagnetic storm, and for recurrent storms a significant increase in this parameter occurs on the 2nd day before the storm's beginning.

\section{Conclusion}

Thus, the fulfilled analysis showed:

1. sporadic and recurrent storms differ by the ratio of the energy characteristics of geomagnetic activity of auroral $\Sigma \mathrm{AE}$ and low-latitude $\Delta \mathrm{Dst}$, each of which has its own linear dependence;

2. the parameter $\boldsymbol{k}=\Delta \mathrm{Dst} / \Sigma \mathrm{AE}$ can be used as a numerical indicator of storm type, it differs significantly for sporadic and recurrent storms: in the XXIII solar cycle average value of $\boldsymbol{k}$ for sporadic storms was 0.0199, and for recurrent storms 0.0076;

3. sporadic storms have a shorter main phase, from 8 to 12 hours, compared with recurrent storms, which have duration of the main phase from 14 to 18 hours;

4. sporadic and recurrent storms differ by their effects on human health;

5. in spite the fact that in the maximum of XXIII solar cycle there were extra storms with an intensity of more than $400 \mathrm{nT}$, the general statistical regularities persist: moderate and intense storms are more often sporadic, and weak ones are recurrent.

Acknowledgements. This work was made in the frame of project of the Program of Complex Scientific Researches in Republic Sakha (Yakutia) 2016- 2020, Russia and partly supported by RFBR grants № 15-45-05090-p_east_a, and № 15-42-05085-p_east_a.

\section{References}

[1] Yu.I. Yermolaev, M.Yu. Yermolaev, Cosmic Investigation, 41:2, 105-109 (2003) (in Russian)

[2] N. Srivastava, P. Venkatakrishnan, J. Geophys. Res., 109, doi:10.1029/2003JA010175 (2004)

[3] J. Zhang et al., J. Geophys. Res., 112, doi:10.1029/2007JA012321 (2007)

[4] E. Echer et al., J. Geophys. Res., 113, doi:10.1029/2007JA012744 (2008)

[5] D. Xu et al., Planetary Space Sci., 57, 1500-1513 (2009)

[6] R.P. Kane, Planetary and Space Sci., 58, 392-400 (2010)

[7] C. Oprea et al., Ann. Geophys., 31, 1285-1295 (2013)

[8] N.S. Nikolaeva, Yu.I. Yermolaev, I.G. Lodkina, Geomagnetism and Aeronomy, 51, 51-67 (2011)

[9] I.G. Richardson, H.V. Cane, Sp. Weather, 9, doi:10.1029/2011SW000670 (2011)

[10] E. Echer, B.T. Tsurutani, W.D. Gonzalez, J. Geophys. Res., 118, 385-392 (2013)

[11] A.A. Petrukovich, Yu.I. Yermolaev, N.A. Aismont, Proc. 11-th conf. "Physics of plasma in solar system", Moskow (2016) (in Russian)

[12] L.P. Shadrina et al., Magnetospheric Phenomena, 13, 33-37 (1989) (in Russian)

[13] L.P. Shadrina, Solar-Terrestrial Physics, 1, 107-108 (2002) (in Russian)

[14] L.P. Shadrina, V.G. Vasileva, Issledovaniya po geomagnetizmu, aeronomii i fizike Solnza, (ISZF SB RAS, Irkutsk), 112, 163-167 (2001)

[15] M.G. Gelberg, S.Z. Kershengolz, S.V. Sharaeva, Geomagnetism and Aeronomy, 40, 26-32 (2000)

[16] S.I. Soloviev et al. Geomagnetism and Aeronomy, 48, 306-319 (2008)

[17] T.I. Pulkkinen et al., Adv. Space Res., 30, 2231-2240 (2002)

[18] L.P. Shadrina, S.N. Samsonov, V.I. Manykina, Yakut Medical Journal, 43, 108-111 (2013) 